\title{
ON THE CAGINALP PHASE-FIELD SYSTEMS WITH TWO TEMPERATURES AND THE MAXWELL-CATTANEO LAW
}

\author{
ALAIN MIRANVILLE ${ }^{1}$, RAMON QUINTANILLA ${ }^{2}$
}

\begin{abstract}
Our aim in this paper is to study generalizations of the nonconserved and conserved Caginalp phase-field systems based on the Maxwell-Cattaneo law with two temperatures for heat conduction. In particular, we obtain well-posedness results and study the dissipativity of the associated solution operators.
\end{abstract}

\section{INTRODUCTION}

G. Caginalp proposed in [7], [8] and [9] two phase-field systems, namely,

$$
\frac{\partial u}{\partial t}-\Delta u+f(u)=T
$$

$$
\frac{\partial T}{\partial t}-\Delta T=-\frac{\partial u}{\partial t}
$$

called nonconserved system, and

$$
\begin{gathered}
\frac{\partial u}{\partial t}+\Delta^{2} u-\Delta f(u)=-\Delta T, \\
\frac{\partial T}{\partial t}-\Delta T=-\frac{\partial u}{\partial t}
\end{gathered}
$$

called conserved system (in the sense that, when endowed with Neumann boundary conditions, the spatial average of $u$ is conserved). In this context, $u$ is the order parameter, $T$ is the relative temperature (defined as $T=\tilde{T}-T_{E}$, where $\tilde{T}$ is the absolute temperature and $T_{E}$ is the equilibrium melting temperature) and $f$ is the derivative of a double-well potential $F$ (a typical choice is $F(s)=\frac{1}{4}\left(s^{2}-1\right)^{2}$, hence the usual cubic nonlinear term $\left.f(s)=s^{3}-s\right)$. Furthermore, we have set all physical parameters equal to one. These systems have been introduced to model phase transition phenomena, such as melting-solidification phenomena, and have been much studied from a mathematical point of view. We refer the reader to, e.g., [1], [2], [3], [4], [5], [6], [13], [14], [15], [20], [21], [22], [23], [24], [25], [26], [31], [33], [40] and [45].

In particular, these two phase-field systems are based on the usual Fourier law for heat conduction,

2010 Mathematics Subject Classification. 35K55, 35J60, 80A22.

Key words and phrases. Caginalp systems, Maxwell-Cattaneo law, two temperatures, well-posedness, dissipativity. 


$$
q=-\nabla T
$$

where $q$ is the heat flux. Now, one essential drawback of the Fourier law is that it predicts that thermal signals propagate at an infinite speed, which violates causality (the so-called paradox of heat conduction, see [17]). To overcome this drawback, or at least to account for more realistic features, several alternatives to the Fourier law, based, e.g., on the Maxwell-Cattaneo law or recent laws from thermomechanics, have been proposed and studied, in the context of the Caginalp phase-field systems, in [28], [29], [32], [34], [35], [36], [37] and [38].

In the late 1960's, several authors proposed a heat conduction theory based on two temperatures (see [10], [11] and [12]). More precisely, one now considers the conductive temperature $T$ and the thermodynamic temperature $\theta$. For time-independent problems, the difference between these temperatures is proportional to the heat supply; they thus coincide when there is no heat supply. However, for time-dependent problems, they are generally different, even in the absence of heat supply: this is in particular the case for non-simple materials. In that case, the two temperatures are related as follows:

$$
\theta=T-\Delta T
$$

and (1.1) and (1.2) should be replaced by

$$
\frac{\partial u}{\partial t}-\Delta u+f(u)=T-\Delta T
$$

and

$$
\frac{\partial u}{\partial t}+\Delta^{2} u-\Delta f(u)=-\Delta(T-\Delta T)
$$

respectively.

The nonconserved system was studied in [18] for the classical Fourier law with two temperatures and in [39] for the type III thermomechanics theory (see [27]) with two temperatures recently proposed in [42] (see also [19]).

In this paper, we consider the theory of two-temperature-generalized thermoelasticity proposed in [44] and based on the Maxwell-Cattaneo law.

In that case, in order to obtain the corresponding generalized heat equation, one writes

$$
\frac{\partial H}{\partial t}=-\operatorname{div} q
$$

where

$$
H=u+T-\Delta T(=u+\theta)
$$

is the enthalpy and the heat flux $q$ satisfies the Maxwell-Cattaneo law (see [44]), 


$$
q+\tau \frac{\partial q}{\partial t}=-\nabla T, \tau>0
$$

In particular, it follows from (1.9) that

$$
\frac{\partial^{2} H}{\partial t^{2}}+\tau \frac{\partial H}{\partial t}=-\operatorname{div}(q+\tau \nabla q)
$$

hence, in view of (1.11),

$$
\frac{\partial^{2} H}{\partial t^{2}}+\tau \frac{\partial H}{\partial t}=\Delta T
$$

We thus deduce from (1.10) and (1.12) the generalized heat equation

$$
(I-\Delta)\left(\frac{\partial^{2} T}{\partial t^{2}}+\tau \frac{\partial T}{\partial t}\right)-\Delta T=-\frac{\partial^{2} u}{\partial t^{2}}-\tau \frac{\partial u}{\partial t} .
$$

Here, the presence of the second derivative $\frac{\partial^{2} u}{\partial t^{2}}$ makes the mathematical analysis of the equation particularly difficult and, to overcome such a difficulty, we will rewrite the equation in a different way, keeping the enthalpy $H$ as unknown. Indeed, it follows from (1.10) and (1.12) that

$$
(I-\Delta)\left(\frac{\partial^{2} H}{\partial t^{2}}+\tau \frac{\partial H}{\partial t}\right)=\Delta(T-\Delta T)
$$

hence

$$
(I-\Delta)\left(\frac{\partial^{2} H}{\partial t^{2}}+\tau \frac{\partial H}{\partial t}\right)-\Delta H=-\Delta u
$$

Furthermore, owing again to (1.10), (1.7) and (1.8) can be rewritten as

$$
\frac{\partial u}{\partial t}-\Delta u+u+f(u)=H
$$

and

$$
\frac{\partial u}{\partial t}+\Delta^{2} u-\Delta u-\Delta f(u)=-\Delta H
$$

respectively.

Our aim in this paper is to study the well-posedness and the dissipativity of (1.14) and (1.15) and of (1.14) and (1.16). For the sake of simplicity, we endow these equations with Dirichlet boundary conditions. Furthermore, when studying the conserved model (1.14) and (1.16), the main difficulty, when compared with the nonconserved model (1.14) and (1.15), is to derive proper $H^{2}$-estimates. 
Notation. We denote by $((\cdot, \cdot))$ the usual $L^{2}$-scalar product, with associated norm $\|\cdot\|$. We further set $((\cdot, \cdot))_{-1}=\left(\left((-\Delta)^{-\frac{1}{2}} \cdot,(-\Delta)^{-\frac{1}{2}} \cdot\right)\right)$, with associated norm $\|\cdot\|_{-1}$, where $(-\Delta)^{-1}$ denotes the inverse minus Laplace operator associated with Dirichlet boundary conditions. Note that $\|\cdot\|_{-1}$ is equivalent to the usual $H^{-1}$-norm on $H^{-1}(\Omega)=H_{0}^{1}(\Omega)^{\prime}$. More generally, $\|\cdot\|_{X}$ denotes the norm on the Banach space $X$.

Throughout the paper, the same letters $c$ and $c^{\prime}$ denote (generally positive) constants which may vary from line to line. Similarly, the same letter $Q$ denotes (positive) monotone increasing (with respect to each argument) and continuous functions which may vary from line to line.

\section{THE NONCONSERVED MODEL}

2.1. Setting of the problem. We consider in this section the following initial and boundary value problem, in a bounded and regular domain $\Omega \subset \mathbb{R}^{N}, N=1,2$ or 3 , with boundary $\Gamma$ :

$$
\begin{gathered}
\frac{\partial u}{\partial t}-\Delta u+u+f(u)=H \\
(I-\Delta)\left(\frac{\partial^{2} H}{\partial t^{2}}+\frac{\partial H}{\partial t}\right)-\Delta H=-\Delta u \\
u=H=0 \text { on } \Gamma \\
\left.u\right|_{t=0}=u_{0},\left.H\right|_{t=0}=H_{0},\left.\frac{\partial H}{\partial t}\right|_{t=0}=H_{1},
\end{gathered}
$$

where, for simplicity, we have set $\tau$ equal to one.

As far as the nonlinear term $f$ is concerned, we assume that

$$
\begin{gathered}
f \in \mathcal{C}^{2}(\mathbb{R}), f(0)=0, \\
f^{\prime} \geq-c_{0}, c_{0} \geq 0, \\
f(s) s \geq c_{1} F(s)-c_{2} \geq-c_{3}, c_{1}>0, c_{2}, c_{3} \geq 0, s \in \mathbb{R},
\end{gathered}
$$

where $F(s)=\int_{0}^{s} f(\xi) d \xi$. In particular, the usual cubic nonlinear term $f(s)=s^{3}-s$ satisfies these assumptions.

Remark 2.1. Actually, in this section, $f \in \mathcal{C}^{1}(\mathbb{R})$ would be sufficient; we will need the higher regularity above in order to deal with the conserved model. 
2.2. A priori estimates. The estimates derived in this subsection are formal, but they can easily be justified within a Galerkin scheme.

We multiply (2.1) by $\frac{\partial u}{\partial t}$ and have, integrating over $\Omega$ and by parts,

$$
\frac{d}{d t}\left(\|u\|_{H^{1}(\Omega)}^{2}+2 \int_{\Omega} F(u) d x\right)+2\left\|\frac{\partial u}{\partial t}\right\|^{2}=2\left(\left(H, \frac{\partial u}{\partial t}\right)\right),
$$

noting that $\|\cdot\|_{H^{1}(\Omega)}^{2}=\|\cdot\|^{2}+\|\nabla \cdot\|^{2}$.

We then multiply $(2.2)$ by $(-\Delta)^{-1} \frac{\partial H}{\partial t}$ to obtain

$$
\frac{d}{d t}\left(\|H\|^{2}+\left\|\frac{\partial H}{\partial t}\right\|_{-1}^{2}+\left\|\frac{\partial H}{\partial t}\right\|^{2}\right)+2\left(\left\|\frac{\partial H}{\partial t}\right\|_{-1}^{2}+\left\|\frac{\partial H}{\partial t}\right\|^{2}\right)=2\left(\left(u, \frac{\partial H}{\partial t}\right)\right) .
$$

Noting that

we finally find, summing (2.8) and (2.9),

$$
\left(\left(H, \frac{\partial u}{\partial t}\right)\right)=\frac{d}{d t}((u, H))-\left(\left(u, \frac{\partial H}{\partial t}\right)\right),
$$

$$
\begin{aligned}
\frac{d}{d t}\left(\|\nabla u\|^{2}\right. & \left.+2 \int_{\Omega} F(u) d x+\|u-H\|^{2}+\left\|\frac{\partial H}{\partial t}\right\|_{-1}^{2}+\left\|\frac{\partial H}{\partial t}\right\|^{2}\right) \\
& +2\left(\left\|\frac{\partial u}{\partial t}\right\|^{2}+\left\|\frac{\partial H}{\partial t}\right\|_{-1}^{2}+\left\|\frac{\partial H}{\partial t}\right\|^{2}\right)=0 .
\end{aligned}
$$

Next, we multiply (2.1) by $u$ and have, owing to (2.7),

$$
\frac{d}{d t}\|u\|^{2}+2\|u\|_{H^{1}(\Omega)}^{2}+c \int_{\Omega} F(u) d x \leq 2((H, u))+c^{\prime} .
$$

Multiplying then $(2.2)$ by $(-\Delta)^{-1} H$, we obtain

$$
\begin{aligned}
\frac{d}{d t}\left(\|H\|_{-1}^{2}+\right. & \left.\|H\|^{2}+2\left(\left(\frac{\partial H}{\partial t}, H\right)\right)_{-1}+2\left(\left(\frac{\partial H}{\partial t}, H\right)\right)\right)+2\|H\|^{2} \\
& =2((H, u))+2\left(\left\|\frac{\partial H}{\partial t}\right\|_{-1}^{2}+\left\|\frac{\partial H}{\partial t}\right\|^{2}\right) .
\end{aligned}
$$

Summing (2.11) and (2.12), we find

$$
\begin{aligned}
& \frac{d}{d t}\left(\|u\|^{2}+\|H\|_{-1}^{2}+\|H\|^{2}+2\left(\left(\frac{\partial H}{\partial t}, H\right)\right)_{-1}+2\left(\left(\frac{\partial H}{\partial t}, H\right)\right)\right)+c\left(\|u-H\|^{2}\right. \\
& \left.\quad+\|\nabla u\|^{2}+2 \int_{\Omega} F(u) d x\right) \leq 2\left(\left\|\frac{\partial H}{\partial t}\right\|_{-1}^{2}+\left\|\frac{\partial H}{\partial t}\right\|^{2}\right)+c^{\prime}, c>0 .
\end{aligned}
$$

Summing finally (2.10) and $\delta_{1}$ times (2.13), where $\delta_{1}>0$ is chosen small enough, we have a differential inequality of the form

$$
\frac{d E_{1}}{d t}+c\left(E_{1}+\left\|\frac{\partial u}{\partial t}\right\|^{2}\right) \leq c^{\prime}, c>0
$$


where

$$
\begin{aligned}
& E_{1}=\|\nabla u\|^{2}+2 \int_{\Omega} F(u) d x+\|u-H\|^{2}+\left\|\frac{\partial H}{\partial t}\right\|_{-1}^{2}+\left\|\frac{\partial H}{\partial t}\right\|^{2} \\
& +\delta_{1}\left(\|u\|^{2}+\|H\|_{-1}^{2}+\|H\|^{2}+2\left(\left(\frac{\partial H}{\partial t}, H\right)\right)_{-1}+2\left(\left(\frac{\partial H}{\partial t}, H\right)\right)\right)
\end{aligned}
$$

satisfies

$$
E_{1} \geq c\left(\|u\|_{H^{1}(\Omega)}^{2}+\int_{\Omega} F(u) d x+\|H\|^{2}+\left\|\frac{\partial H}{\partial t}\right\|^{2}\right)-c^{\prime}, c>0 .
$$

We now multiply $(2.2)$ by $\frac{\partial H}{\partial t}$ to obtain

$$
\frac{d}{d t}\left(\|\nabla H\|^{2}+\left\|\frac{\partial H}{\partial t}\right\|_{H^{1}(\Omega)}^{2}\right)+\left\|\frac{\partial H}{\partial t}\right\|_{H^{1}(\Omega)}^{2} \leq\|\nabla u\|^{2} .
$$

Multiplying also $(2.2)$ by $H$, we find

$$
\frac{d}{d t}\left(\|H\|_{H^{1}(\Omega)}^{2}+2\left(\left(\frac{\partial H}{\partial t}, H\right)\right)+2\left(\left(\nabla \frac{\partial H}{\partial t}, \nabla H\right)\right)\right)+\|\nabla H\|^{2} \leq\|\nabla u\|^{2}+2\left\|\frac{\partial H}{\partial t}\right\|_{H^{1}(\Omega)}^{2} .
$$

Summing (2.14), $\delta_{2}$ times (2.17) and $\delta_{3}$ times (2.18), where $\delta_{2}, \delta_{3}>0$ are chosen small enough, we have a differential inequality of the form

$$
\frac{d E_{2}}{d t}+c\left(E_{2}+\left\|\frac{\partial u}{\partial t}\right\|^{2}\right) \leq c^{\prime}, c>0
$$

where

$$
\begin{gathered}
E_{2}=E_{1}+\delta_{2}\left(\|\nabla H\|^{2}+\left\|\frac{\partial H}{\partial t}\right\|_{H^{1}(\Omega)}^{2}\right) \\
+\delta_{3}\left(\|H\|_{H^{1}(\Omega)}^{2}+2\left(\left(\frac{\partial H}{\partial t}, H\right)\right)+2\left(\left(\nabla \frac{\partial H}{\partial t}, \nabla H\right)\right)\right)
\end{gathered}
$$

satisfies

$$
E_{2} \geq c\left(\|u\|_{H^{1}(\Omega)}^{2}+\int_{\Omega} F(u) d x+\|H\|_{H^{1}(\Omega)}^{2}+\left\|\frac{\partial H}{\partial t}\right\|_{H^{1}(\Omega)}^{2}\right)-c^{\prime}, c>0 .
$$

We finally multiply (2.1) by $-\Delta u$ and obtain, owing to (2.6) and classical elliptic regularity results,

$$
\frac{d}{d t}\|\nabla u\|^{2}+c\|u\|_{H^{2}(\Omega)}^{2} \leq c^{\prime}\left(\|\nabla u\|^{2}+\|H\|^{2}\right), c>0 .
$$

Summing (2.20) and $\delta_{4}$ times (2.22), where $\delta_{4}>0$ is chosen small enough, we find a differential inequality of the form 


$$
\frac{d E_{3}}{d t}+c\left(E_{3}+\|u\|_{H^{2}(\Omega)}^{2}+\left\|\frac{\partial u}{\partial t}\right\|^{2}\right) \leq c^{\prime}, c>0
$$

where

$$
E_{3}=E_{2}+\delta_{4}\|\nabla u\|^{2}
$$

satisfies

$$
E_{3} \geq c\left(\|u\|_{H^{1}(\Omega)}^{2}+\int_{\Omega} F(u) d x+\|H\|_{H^{1}(\Omega)}^{2}+\left\|\frac{\partial H}{\partial t}\right\|_{H^{1}(\Omega)}^{2}\right)-c^{\prime}, c>0 .
$$

In a second step, we differentiate (2.1) with respect to time to have the initial and boundary value problem

$$
\begin{gathered}
\frac{\partial}{\partial t} \frac{\partial u}{\partial t}-\Delta \frac{\partial u}{\partial t}+\frac{\partial u}{\partial t}+f^{\prime}(u) \frac{\partial u}{\partial t}=\frac{\partial H}{\partial t} \\
\frac{\partial u}{\partial t}=0 \text { on } \Gamma \\
\frac{\partial u}{\partial t}(0)=\Delta u_{0}-u_{0}-f\left(u_{0}\right)+H_{0} .
\end{gathered}
$$

Note that, if $u_{0} \in H^{2}(\Omega) \cap H_{0}^{1}(\Omega)$ and $H_{0} \in L^{2}(\Omega)$, then $\frac{\partial u}{\partial t}(0) \in L^{2}(\Omega)$ and

$$
\left\|\frac{\partial u}{\partial t}(0)\right\| \leq Q\left(\left\|u_{0}\right\|_{H^{2}(\Omega)},\left\|H_{0}\right\|\right) .
$$

Indeed, it follows from the continuity of $f$ and the continuous embedding $H^{2}(\Omega) \subset \mathcal{C}(\bar{\Omega})$ that $\left\|f\left(u_{0}\right)\right\| \leq Q\left(\left\|u_{0}\right\|_{H^{2}(\Omega)}\right)$.

Multiplying $(2.26)$ by $\frac{\partial u}{\partial t}$, we obtain, in view of (2.6),

$$
\frac{d}{d t}\left\|\frac{\partial u}{\partial t}\right\|^{2}+c\left\|\frac{\partial u}{\partial t}\right\|_{H^{1}(\Omega)}^{2} \leq c^{\prime}\left(\left\|\frac{\partial u}{\partial t}\right\|^{2}+\left\|\frac{\partial H}{\partial t}\right\|^{2}\right), c>0 .
$$

Summing then (2.23) and $\delta_{5}$ times $(2.30)$, where $\delta_{5}>0$ is chosen small enough, we find a differential inequality of the form

$$
\frac{d E_{4}}{d t}+c\left(E_{4}+\|u\|_{H^{2}(\Omega)}^{2}+\left\|\frac{\partial u}{\partial t}\right\|_{H^{1}(\Omega)}^{2}\right) \leq c^{\prime}, c>0
$$

where

$$
E_{4}=E_{3}+\delta_{5}\left\|\frac{\partial u}{\partial t}\right\|^{2}
$$

satisfies 


$$
E_{4} \geq c\left(\|u\|_{H^{1}(\Omega)}^{2}+\int_{\Omega} F(u) d x+\left\|\frac{\partial u}{\partial t}\right\|^{2}+\|H\|_{H^{1}(\Omega)}^{2}+\left\|\frac{\partial H}{\partial t}\right\|_{H^{1}(\Omega)}^{2}\right)-c^{\prime}, c>0 .
$$

We finally rewrite (2.1) as an elliptic equation, for $t>0$ fixed,

$$
-\Delta u+u+f(u)=-\frac{\partial u}{\partial t}+H, u=0 \text { on } \Gamma .
$$

Multiplying (2.34) by $-\Delta u$, we have, owing to (2.6),

$$
\|\Delta u\|^{2} \leq c\left(\|\nabla u\|^{2}+\left\|\frac{\partial u}{\partial t}\right\|^{2}+\|H\|^{2}\right),
$$

hence, owing to classical elliptic regularity results,

$$
\|u(t)\|_{H^{2}(\Omega)}^{2} \leq c E_{4}(t), t \geq 0 .
$$

Having this, we multiply $(2.2)$ by $-\Delta \frac{\partial H}{\partial t}$ and $-\Delta H$ to obtain

$$
\frac{d}{d t}\left(\|\Delta H\|^{2}+\left\|\nabla \frac{\partial H}{\partial t}\right\|^{2}+\left\|\Delta \frac{\partial H}{\partial t}\right\|^{2}\right)+\left\|\nabla \frac{\partial H}{\partial t}\right\|^{2}+\left\|\Delta \frac{\partial H}{\partial t}\right\|^{2} \leq\|\Delta u\|^{2}
$$

and

$$
\begin{aligned}
\frac{d}{d t}\left(\|\nabla H\|^{2}+\right. & \left.\|\Delta H\|^{2}+2\left(\left(\nabla \frac{\partial H}{\partial t}, \nabla H\right)\right)+2\left(\left(\Delta \frac{\partial H}{\partial t}, \Delta H\right)\right)\right)+\|\Delta H\|^{2} \\
& \leq\|\Delta u\|^{2}+2\left(\left\|\nabla \frac{\partial H}{\partial t}\right\|^{2}+\left\|\Delta \frac{\partial H}{\partial t}\right\|^{2}\right),
\end{aligned}
$$

respectively. Summing (2.36) and $\delta_{6}$ times (2.37), where $\delta_{6}>0$ is chosen small enough, we find, in view of (2.35), a differential inequality of the form

$$
\frac{d E_{5}}{d t}+c E_{5} \leq c^{\prime} E_{4}, c>0
$$

where

$$
\begin{gathered}
E_{5}=\|\Delta H\|^{2}+\left\|\nabla \frac{\partial H}{\partial t}\right\|^{2}+\left\|\Delta \frac{\partial H}{\partial t}\right\|^{2} \\
+\delta_{6}\left(\|\nabla H\|^{2}+\|\Delta H\|^{2}+2\left(\left(\nabla \frac{\partial H}{\partial t}, \nabla H\right)\right)+2\left(\left(\Delta \frac{\partial H}{\partial t}, \Delta H\right)\right)\right)
\end{gathered}
$$

satisfies

$$
E_{5} \geq c\left(\|H\|_{H^{2}(\Omega)}^{2}+\left\|\frac{\partial H}{\partial t}\right\|_{H^{2}(\Omega)}^{2}\right), c>0
$$


2.3. The dissipative semigroup. We have the

Theorem 2.2. We assume that (2.5)-(2.7) hold. Then, for every $\left(u_{0}, H_{0}, H_{1}\right) \in\left(H^{2}(\Omega) \cap\right.$ $\left.H_{0}^{1}(\Omega)\right)^{3},(2.1)-(2.4)$ possesses a unique solution $\left(u, H, \frac{\partial H}{\partial t}\right)$ such that

and

$$
\left(u, H, \frac{\partial H}{\partial t}\right) \in L^{\infty}\left(\mathbb{R}^{+} ; H^{2}(\Omega) \cap H_{0}^{1}(\Omega)\right)^{3}
$$

$$
\frac{\partial u}{\partial t} \in L^{\infty}\left(\mathbb{R}^{+} ; L^{2}(\Omega)\right) \cap L^{2}\left(0, T ; H_{0}^{1}(\Omega)\right), \forall T>0
$$

Proof. The proof of existence is based on the a priori estimates derived in the previous subsection and, e.g., a standard Galerkin scheme.

In particular, it follows from (2.31) and Gronwall's lemma that

$$
E_{4}(t) \leq e^{-c t} E_{4}(0)+c^{\prime}, c>0, t \geq 0
$$

which yields, owing to (2.33), the continuity of $f$ and the continuous embedding $H^{2}(\Omega) \subset$ $\mathcal{C}(\bar{\Omega})$

$$
\begin{gathered}
\|u(t)\|_{H^{1}(\Omega)}^{2}+\left\|\frac{\partial u}{\partial t}(t)\right\|^{2}+\|H(t)\|_{H^{1}(\Omega)}^{2}+\left\|\frac{\partial H}{\partial t}(t)\right\|_{H^{1}(\Omega)}^{2} \\
\leq e^{-c t} Q\left(\left\|u_{0}\right\|_{H^{2}(\Omega)}^{2},\left\|H_{0}\right\|_{H^{1}(\Omega)}^{2},\left\|H_{1}\right\|_{H^{1}(\Omega)}^{2}\right)+c^{\prime}, c>0, t \geq 0 .
\end{gathered}
$$

It then follows from (2.35) and (2.41)-(2.42) that

$$
\|u(t)\|_{H^{2}(\Omega)}^{2} \leq e^{-c t} Q\left(\left\|u_{0}\right\|_{H^{2}(\Omega)}^{2},\left\|H_{0}\right\|_{H^{1}(\Omega)}^{2},\left\|H_{1}\right\|_{H^{1}(\Omega)}^{2}\right)+c^{\prime}, c>0, t \geq 0,
$$

and from (2.38), (2.40), (2.41)-(2.42) and Gronwall's lemma that

$$
\begin{gathered}
\|H(t)\|_{H^{2}(\Omega)}^{2}+\left\|\frac{\partial H}{\partial t}(t)\right\|_{H^{2}(\Omega)}^{2} \\
\leq e^{-c t} Q\left(\left\|u_{0}\right\|_{H^{2}(\Omega)}^{2},\left\|H_{0}\right\|_{H^{2}(\Omega)}^{2},\left\|H_{1}\right\|_{H^{2}(\Omega)}^{2}\right)+c^{\prime}, c>0, t \geq 0 .
\end{gathered}
$$

Let now $\left(u^{(1)}, H^{(1)}, \frac{\partial H^{(1)}}{\partial t}\right)$ and $\left(u^{(2)}, H^{(2)}, \frac{\partial H^{(2)}}{\partial t}\right)$ be two solutions to (2.1)-(2.3) with initial data $\left(u_{0}^{(1)}, H_{0}^{(1)}, H_{1}^{(1)}\right)$ and $\left(u_{0}^{(2)}, H_{0}^{(2)}, H_{1}^{(2)}\right)$, respectively. We set

and

$$
\left(u, H, \frac{\partial H}{\partial t}\right)=\left(u^{(1)}, H^{(1)}, \frac{\partial H^{(1)}}{\partial t}\right)-\left(u^{(2)}, H^{(2)}, \frac{\partial H^{(2)}}{\partial t}\right)
$$

$$
\left(u_{0}, H_{0}, H_{1}\right)=\left(u_{0}^{(1)}, H_{0}^{(1)}, H_{1}^{(1)}\right)-\left(u_{0}^{(2)}, H_{0}^{(2)}, H_{1}^{(2)}\right)
$$

and have

$$
\frac{\partial u}{\partial t}-\Delta u+u+f\left(u^{(1)}\right)-f\left(u^{(2)}\right)=H
$$




$$
\begin{gathered}
(I-\Delta)\left(\frac{\partial^{2} H}{\partial t^{2}}+\frac{\partial H}{\partial t}\right)-\Delta H=-\Delta u, \\
u=H=0 \text { on } \Gamma \\
\left.u\right|_{t=0}=u_{0},\left.H\right|_{t=0}=H_{0},\left.\frac{\partial H}{\partial t}\right|_{t=0}=H_{1} .
\end{gathered}
$$

Multiplying (2.45) by $u$, we obtain, in view of (2.6),

$$
\frac{d}{d t}\|u\|^{2}+\|u\|_{H^{1}(\Omega)}^{2} \leq c\left(\|u\|^{2}+\|H\|^{2}\right) .
$$

Multiplying then $(2.46)$ by $(-\Delta)^{-1} \frac{\partial H}{\partial t}$, we find

$$
\frac{d}{d t}\left(\|H\|^{2}+\left\|\frac{\partial H}{\partial t}\right\|_{-1}^{2}+\left\|\frac{\partial H}{\partial t}\right\|^{2}\right)+\left\|\frac{\partial H}{\partial t}\right\|_{-1}^{2}+\left\|\frac{\partial H}{\partial t}\right\|^{2} \leq\|u\|^{2} .
$$

Summing finally (2.49) and (2.50), we have a differential inequality of the form

$$
\frac{d E_{6}}{d t} \leq c E_{6}
$$

where

$$
E_{6}=\|u\|^{2}+\|H\|^{2}+\left\|\frac{\partial H}{\partial t}\right\|_{-1}^{2}+\left\|\frac{\partial H}{\partial t}\right\|^{2}
$$

satisfies

$$
E_{6} \geq c\left(\|u\|^{2}+\|H\|^{2}+\left\|\frac{\partial H}{\partial t}\right\|^{2}\right), c>0
$$

It thus follows from (2.51)-(2.53) and Gronwall's lemma that

$$
\|u(t)\|^{2}+\|H(t)\|^{2}+\left\|\frac{\partial H}{\partial t}(t)\right\|^{2} \leq c e^{c^{\prime} t}\left(\left\|u_{0}\right\|^{2}+\left\|H_{0}\right\|^{2}+\left\|H_{1}\right\|^{2}\right), t \geq 0,
$$

hence the uniqueness, as well as the continuity with respect to the initial data in the $L^{2}$-norm.

It follows from Theorem 2.2 that we can define the family of solving operators

$$
S(t): \Phi \rightarrow \Phi,\left(u_{0}, H_{0}, H_{1}\right) \mapsto\left(u(t), H(t), \frac{\partial H}{\partial t}(t)\right), t \geq 0
$$

where $\Phi=\left(H^{2}(\Omega) \cap H_{0}^{1}(\Omega)\right)^{3}$. Furthermore, this family of solving operators forms a semigroup, i.e., $S(0)=I$ and $S(t+\tau)=S(t) \circ S(\tau), \forall t, \tau \geq 0$, which is continuous with respect to the $L^{2}$-topology. 
Finally, it follows from (2.42)-(2.44) that we have the

Theorem 2.3. The semigroup $S(t)$ is dissipative in $\Phi$, in the sense that it possesses a bounded absorbing set $\mathcal{B}_{0} \subset \Phi$ (i.e., $\forall B \subset \Phi$ bounded, $\exists t_{0}=t_{0}(B)$ such that $t \geq t_{0}$ implies $\left.S(t) B \subset \mathcal{B}_{0}\right)$.

Remark 2.4. The dissipativity is a first step in view of the study of the (temporal) asymptotic behavior of the associated dynamical system. In particular, an important issue is to prove the existence of finite-dimensional attractors: such objects describe all possible dynamics of the system; furthermore, the finite-dimensionality means, very roughly speaking, that, even though the initial phase space $\Phi$ has infinite dimension, the reduced dynamics can be described by a finite number of parameters (we refer the interested reader to, e.g., [41] and [43] for discussions on this subject). This will be studied elsewhere.

\section{THE CONSERVED MODEL}

We now consider the following initial and boundary value problem:

$$
\begin{gathered}
(-\Delta)^{-1} \frac{\partial u}{\partial t}-\Delta u+u+f(u)=H \\
(I-\Delta)\left(\frac{\partial^{2} H}{\partial t^{2}}+\frac{\partial H}{\partial t}\right)-\Delta H=-\Delta u \\
u=H=0 \text { on } \Gamma \\
\left.u\right|_{t=0}=u_{0},\left.H\right|_{t=0}=H_{0},\left.\frac{\partial H}{\partial t}\right|_{t=0}=H_{1} .
\end{gathered}
$$

As far as the nonlinear term $f$ is concerned, we still assume that (2.5)-(2.7) hold.

3.1. A priori estimates. Here, we can repeat the first estimates made in the previous section for the nonconserved problem, the only difference being that we have a lower regularity on $\frac{\partial u}{\partial t}$, due to the presence of the operator $(-\Delta)^{-1}$. In particular, we have a differential inequality of the form

$$
\frac{d E_{7}}{d t}+c\left(E_{7}+\|u\|_{H^{2}(\Omega)}^{2}+\left\|\frac{\partial u}{\partial t}\right\|_{-1}^{2}\right) \leq c^{\prime}, c>0
$$

where

$$
\begin{gathered}
E_{7}=\|\nabla u\|^{2}+2 \int_{\Omega} F(u) d x+\|u-H\|^{2}+\left\|\frac{\partial H}{\partial t}\right\|_{-1}^{2}+\left\|\frac{\partial H}{\partial t}\right\|^{2} \\
+\delta_{7}\left(\|u\|_{-1}^{2}+\|H\|_{-1}^{2}+\|H\|^{2}+2\left(\left(\frac{\partial H}{\partial t}, H\right)\right)_{-1}+2\left(\left(\frac{\partial H}{\partial t}, H\right)\right)\right) \\
+\delta_{8}\left(\|\nabla H\|^{2}+\left\|\frac{\partial H}{\partial t}\right\|_{H^{1}(\Omega)}^{2}\right)
\end{gathered}
$$


satisfies

$$
+\delta_{9}\left(\|H\|_{H^{1}(\Omega)}^{2}+2\left(\left(\frac{\partial H}{\partial t}, H\right)\right)+2\left(\left(\nabla \frac{\partial H}{\partial t}, \nabla H\right)\right)\right)+\delta_{10}\|u\|^{2}
$$

$$
E_{7} \geq c\left(\|u\|_{H^{1}(\Omega)}^{2}+\int_{\Omega} F(u) d x+\|H\|_{H^{1}(\Omega)}^{2}+\left\|\frac{\partial H}{\partial t}\right\|_{H^{1}(\Omega)}^{2}\right)-c^{\prime}, c>0,
$$

$\delta_{7}, \delta_{8}, \delta_{9}, \delta_{10}>0$ having been chosen small enough.

The next step is to derive $H^{2}$-estimates. Note that, if we rewrite (3.1) as an elliptic equation, for $t>0$ fixed,

$$
-\Delta u+u+f(u)=-(-\Delta)^{-1} \frac{\partial u}{\partial t}+H, u=0 \text { on } \Gamma,
$$

and wish to proceed as in the previous section for the nonconserved model, we need to have an $L^{\infty}\left(L^{2}\right)$-regularity on $(-\Delta)^{-1} \frac{\partial u}{\partial t}$. We now differentiate (3.1) with respect to time to obtain

$$
\begin{gathered}
(-\Delta)^{-1} \frac{\partial}{\partial t} \frac{\partial u}{\partial t}-\Delta \frac{\partial u}{\partial t}+\frac{\partial u}{\partial t}+f^{\prime}(u) \frac{\partial u}{\partial t}=\frac{\partial H}{\partial t}, \\
\frac{\partial u}{\partial t}=0 \text { on } \Gamma, \\
\frac{\partial u}{\partial t}(0)=-\Delta^{2} u_{0}+\Delta u_{0}+\Delta f\left(u_{0}\right)-\Delta H_{0} .
\end{gathered}
$$

Multiplying (3.9) by $\frac{\partial u}{\partial t}$, we find, in view of (2.6),

$$
\frac{1}{2} \frac{d}{d t}\left\|\frac{\partial u}{\partial t}\right\|_{-1}^{2}+\left\|\frac{\partial u}{\partial t}\right\|_{H^{1}(\Omega)}^{2} \leq c_{0}\left\|\frac{\partial u}{\partial t}\right\|^{2}+\left(\left(\frac{\partial H}{\partial t}, \frac{\partial u}{\partial t}\right)\right),
$$

which yields, employing the interpolation inequality

the differential inequality

$$
\left\|\frac{\partial u}{\partial t}\right\|^{2} \leq c\left\|\frac{\partial u}{\partial t}\right\|_{-1}\left\|\frac{\partial u}{\partial t}\right\|_{H^{1}(\Omega)}
$$

$$
\frac{d}{d t}\left\|\frac{\partial u}{\partial t}\right\|_{-1}^{2}+c\left\|\frac{\partial u}{\partial t}\right\|_{H^{1}(\Omega)}^{2} \leq c^{\prime}\left(\left\|\frac{\partial H}{\partial t}\right\|^{2}+\left\|\frac{\partial u}{\partial t}\right\|_{-1}^{2}\right) .
$$

This yields an $L^{\infty}\left(L^{2}\right)$-regularity on $(-\Delta)^{-1} \frac{\partial u}{\partial t}$, provided that $(-\Delta)^{-\frac{1}{2}} \frac{\partial u}{\partial t}(0) \in L^{2}(\Omega)$, which, in view of (3.11), essentially means that $u_{0} \in H^{3}(\Omega) \cap H_{0}^{1}(\Omega)$. This is not satisfactory, in particular, in view of the study of the dissipativity and the existence of (finite-dimensional) attractors.

Actually, we can prove, by proceeding in a more careful way, that $u_{0} \in H^{2}(\Omega) \cap H_{0}^{1}(\Omega)$ suffices, which is indeed what one would expect.

To do so, we first multiply (3.1) by $-\Delta \frac{\partial u}{\partial t}$ to have 


$$
\frac{d}{d t}\|\Delta u\|^{2}+\left\|\frac{\partial u}{\partial t}\right\|^{2} \leq c\left(\|\Delta f(u)\|^{2}+\left\|\Delta \frac{\partial H}{\partial t}\right\|^{2}\right) .
$$

Furthermore, proceeding as in the previous section, we obtain a differential inequality of the form

$$
\frac{d E_{8}}{d t}+c E_{8} \leq c^{\prime}\|\Delta u\|^{2}, c>0
$$

where

$$
\begin{gathered}
E_{8}=\|\Delta H\|^{2}+\left\|\nabla \frac{\partial H}{\partial t}\right\|^{2}+\left\|\Delta \frac{\partial H}{\partial t}\right\|^{2} \\
+\delta_{11}\left(\|\nabla H\|^{2}+\|\Delta H\|^{2}+2\left(\left(\nabla \frac{\partial H}{\partial t}, \nabla H\right)\right)+2\left(\left(\Delta \frac{\partial H}{\partial t}, \Delta H\right)\right)\right)
\end{gathered}
$$

satisfies

$$
E_{8} \geq c\left(\|H\|_{H^{2}(\Omega)}^{2}+\left\|\frac{\partial H}{\partial t}\right\|_{H^{2}(\Omega)}^{2}\right), c>0,
$$

$\delta_{11}>0$ having been chosen small enough. Summing finally (3.13) and $\delta_{12}$ times (3.14), where $\delta_{12}>0$ is chosen small enough, we find a differential inequality of the form

$$
\frac{d E_{9}}{d t} \leq Q\left(\|\Delta u\|^{2}+\left\|\Delta \frac{\partial H}{\partial t}\right\|^{2}\right)
$$

where

$$
E_{9}=\|\Delta u\|^{2}+E_{8}
$$

satisfies

$$
E_{9} \geq c\left(\|u\|_{H^{2}(\Omega)}^{2}+\|H\|_{H^{2}(\Omega)}^{2}+\left\|\frac{\partial H}{\partial t}\right\|_{H^{2}(\Omega)}^{2}\right), c>0 .
$$

Here, we have used the fact that $f$ is of class $\mathcal{C}^{2}$ and the continuous embedding $H^{2}(\Omega) \subset$ $\mathcal{C}(\bar{\Omega})$ once more to deduce that $\|\Delta f(u)\|^{2} \leq Q\left(\|\Delta u\|^{2}\right)$.

Setting $y=\|\Delta u\|^{2}+E_{8}$, we thus have the differential inequality

$$
y^{\prime} \leq Q(y)
$$

Let $z$ be solution to the ODE

$$
z^{\prime}=Q(z), z(0)=y(0)
$$

It follows from the comparison principle that there exists

$$
T_{0}=T_{0}\left(\left\|u_{0}\right\|_{H^{2}(\Omega)},\left\|H_{0}\right\|_{H^{2}(\Omega)},\left\|H_{1}\right\|_{H^{2}(\Omega)}\right)
$$


belonging to, say, $(0,1)$ such that

$$
y(t) \leq z(t), t \in\left[0, T_{0}\right]
$$

so that, in particular,

$$
\|u(t)\|_{H^{2}(\Omega)} \leq Q\left(\left\|u_{0}\right\|_{H^{2}(\Omega)},\left\|H_{0}\right\|_{H^{2}(\Omega)},\left\|H_{1}\right\|_{H^{2}(\Omega)}\right), t \in\left[0, T_{0}\right] .
$$

Next, we multiply $(3.9)$ by $t \frac{\partial u}{\partial t}$ to obtain, proceeding as above,

$$
\frac{d}{d t}\left(t\left\|\frac{\partial u}{\partial t}\right\|_{-1}^{2}\right) \leq c t\left(\left\|\frac{\partial H}{\partial t}\right\|^{2}+\left\|\frac{\partial u}{\partial t}\right\|_{-1}^{2}\right)+\left\|\frac{\partial u}{\partial t}\right\|_{-1}^{2} .
$$

It thus follows from (3.5)-(3.7) (which yield proper estimates on $\left\|\frac{\partial u}{\partial t}\right\|_{-1}^{2}$ and $\left\|\frac{\partial H}{\partial t}\right\|^{2}$ ), (3.24) and Gronwall's lemma that

$$
\left\|\frac{\partial u}{\partial t}\left(T_{0}\right)\right\|_{-1}^{2} \leq Q\left(\left\|u_{0}\right\|_{H^{2}(\Omega)},\left\|H_{0}\right\|_{H^{1}(\Omega)},\left\|H_{1}\right\|_{H^{1}(\Omega)}\right) .
$$

Here, we have used the fact that, since $F$ is continuous, then, owing to the continuous embedding $H^{2}(\Omega) \subset \mathcal{C}(\bar{\Omega})$,

$$
E_{7}(0) \leq Q\left(\left\|u_{0}\right\|_{H^{2}(\Omega)},\left\|H_{0}\right\|_{H^{1}(\Omega)},\left\|H_{1}\right\|_{H^{1}(\Omega)}\right) .
$$

Having this, it follows from (3.5)-(3.7), (3.12) and again Gronwall's lemma that

$$
\left\|\frac{\partial u}{\partial t}(t)\right\|_{-1}^{2} \leq e^{c\left(t-T_{0}\right)} Q\left(\left\|u_{0}\right\|_{H^{2}(\Omega)},\left\|H_{0}\right\|_{H^{1}(\Omega)},\left\|H_{1}\right\|_{H^{1}(\Omega)}\right)\left(1+\left\|\frac{\partial u}{\partial t}\left(T_{0}\right)\right\|_{-1}^{2}\right), t \geq T_{0},
$$

hence, in view of $(3.25)$,

$$
\left\|\frac{\partial u}{\partial t}(t)\right\|_{-1}^{2} \leq e^{c t} Q\left(\left\|u_{0}\right\|_{H^{2}(\Omega)},\left\|H_{0}\right\|_{H^{1}(\Omega)},\left\|H_{1}\right\|_{H^{1}(\Omega)}\right), t \geq T_{0} .
$$

We finally multiply the elliptic equation (3.8) by $-\Delta u$ and find, in view of (2.6),

$$
\|\Delta u(t)\|^{2} \leq c\left(\left\|\frac{\partial u}{\partial t}(t)\right\|_{-1}^{2}+\|H(t)\|^{2}\right), t \geq T_{0},
$$

hence, in view of (3.5)-(3.7), (3.27) and standard elliptic regularity results,

$$
\|u(t)\|_{H^{2}(\Omega)} \leq e^{c t} Q\left(\left\|u_{0}\right\|_{H^{2}(\Omega)},\left\|H_{0}\right\|_{H^{1}(\Omega)},\left\|H_{1}\right\|_{H^{1}(\Omega)}\right), t \geq T_{0} .
$$

Actually, there holds, in view of (3.23),

$$
\|u(t)\|_{H^{2}(\Omega)} \leq e^{c t} Q\left(\left\|u_{0}\right\|_{H^{2}(\Omega)},\left\|H_{0}\right\|_{H^{2}(\Omega)},\left\|H_{1}\right\|_{H^{2}(\Omega)}\right), t \geq 0 .
$$

Note that this estimate is not dissipative and a priori grows as $t \rightarrow+\infty$.

In order to derive a dissipative estimate, we now multiply (3.1) by $-\Delta u$ and have, owing to (2.6), 


$$
\frac{d}{d t}\|u\|^{2}+\|\Delta u\|^{2}+\|\nabla u\|^{2} \leq 2 c_{0}\|\nabla u\|^{2}+\|H\|^{2} .
$$

Integrating (3.31) over $(0,1)$ and employing (3.5)-(3.7) to estimate $\|\nabla u\|^{2}$ and $\|H\|^{2}$, we have

$$
\int_{0}^{1}\|\Delta u\|^{2} d x \leq c E_{7}(0)+c^{\prime}
$$

which yields that there exists $T \in(0,1)$ such that

$$
\|u(T)\|_{H^{2}(\Omega)}^{2} \leq c E_{7}(0)+c^{\prime} .
$$

Actually, repeating the above estimates (and, in particular, employing (3.30)), but starting from $t=T$ instead of $t=0$, we obtain the inequality

$$
\|u(1)\|_{H^{2}(\Omega)}^{2} \leq Q\left(E_{7}(0)\right) .
$$

Repeating again the above estimates (i.e., those leading to (3.33)), we find, for $t \geq 1$,

$$
\|u(t)\|_{H^{2}(\Omega)}^{2} \leq Q\left(E_{7}(t-1)\right),
$$

where the function $Q$ does not depend on $t$ (note indeed that (3.21) is an autonomous ODE; actually, here, $Q$ is the same function as in (3.33)). Employing once more (3.5)-(3.7) and Gronwall's lemma, we finally deduce that

$$
\|u(t)\|_{H^{2}(\Omega)}^{2} \leq e^{-c t} Q\left(\left\|u_{0}\right\|_{H^{2}(\Omega)},\left\|H_{0}\right\|_{H^{1}(\Omega)},\left\|H_{1}\right\|_{H^{1}(\Omega)}\right)+c^{\prime}, t \geq 1,
$$

hence a dissipative estimate.

Dissipative estimates on the $H^{2}$-norms of $H$ and $\frac{\partial H}{\partial t}$ then follow from (3.14), as in the previous section.

3.2. The dissipative semigroup. We have the

Theorem 3.1. We assume that (2.5)-(2.7) hold. Then, for every $\left(u_{0}, H_{0}, H_{1}\right) \in\left(H^{2}(\Omega) \cap\right.$ $\left.H_{0}^{1}(\Omega)\right)^{3}$, (3.1)-(3.4) possesses a unique solution $\left(u, H, \frac{\partial H}{\partial t}\right)$ such that

and

$$
\left(u, H, \frac{\partial H}{\partial t}\right) \in L^{\infty}\left(\mathbb{R}^{+} ; H^{2}(\Omega) \cap H_{0}^{1}(\Omega)\right)^{3}
$$

$$
\frac{\partial u}{\partial t} \in L^{2}\left(0, T ; H^{-1}(\Omega)\right), \forall T>0 .
$$

Proof. The proof of existence is again based on the a priori estimates derived in the previous subsection and, e.g., a standard Galerkin scheme.

Let now $\left(u^{(1)}, H^{(1)}, \frac{\partial H^{(1)}}{\partial t}\right)$ and $\left(u^{(2)}, H^{(2)}, \frac{\partial H^{(2)}}{\partial t}\right)$ be two solutions to (3.1)-(3.3) with initial data $\left(u_{0}^{(1)}, H_{0}^{(1)}, H_{1}^{(1)}\right)$ and $\left(u_{0}^{(2)}, H_{0}^{(2)}, H_{1}^{(2)}\right)$, respectively. Setting again 
and

$$
\left(u, H, \frac{\partial H}{\partial t}\right)=\left(u^{(1)}, H^{(1)}, \frac{\partial H^{(1)}}{\partial t}\right)-\left(u^{(2)}, H^{(2)}, \frac{\partial H^{(2)}}{\partial t}\right)
$$

$$
\left(u_{0}, H_{0}, H_{1}\right)=\left(u_{0}^{(1)}, H_{0}^{(1)}, H_{1}^{(1)}\right)-\left(u_{0}^{(2)}, H_{0}^{(2)}, H_{1}^{(2)}\right),
$$

we have

$$
\begin{gathered}
(-\Delta)^{-1} \frac{\partial u}{\partial t}-\Delta u+u+f\left(u^{(1)}\right)-f\left(u^{(2)}\right)=H, \\
(I-\Delta)\left(\frac{\partial^{2} H}{\partial t^{2}}+\frac{\partial H}{\partial t}\right)-\Delta H=-\Delta u, \\
u=H=0 \text { on } \Gamma, \\
\left.u\right|_{t=0}=u_{0},\left.H\right|_{t=0}=H_{0},\left.\frac{\partial H}{\partial t}\right|_{t=0}=H_{1} .
\end{gathered}
$$

Procceding as in the proof of Theorem 2.2, we obtain a differential inequality of the form

$$
\frac{d E_{10}}{d t}+\|u\|_{H^{1}(\Omega)}^{2} \leq c\left(\|u\|^{2}+\|H\|^{2}\right)
$$

where

$$
E_{10}=\|u\|_{-1}^{2}+\|H\|^{2}+\left\|\frac{\partial H}{\partial t}\right\|_{-1}^{2}+\left\|\frac{\partial H}{\partial t}\right\|^{2}
$$

satisfies

$$
E_{10} \geq c\left(\|u\|_{-1}^{2}+\|H\|^{2}+\left\|\frac{\partial H}{\partial t}\right\|^{2}\right), c>0
$$

Using finally the interpolation inequality

we find the differential inequality

$$
\|u\|^{2} \leq c\|u\|_{-1}\|u\|_{H^{1}(\Omega)},
$$

$$
\frac{d E_{10}}{d t} \leq c E_{10}
$$

hence, owing to (3.42)-(3.43) and Gronwall's lemma,

$$
\|u(t)\|_{-1}^{2}+\|H(t)\|^{2}+\left\|\frac{\partial H}{\partial t}(t)\right\|^{2} \leq c e^{c^{\prime} t}\left(\left\|u_{0}\right\|_{-1}^{2}+\left\|H_{0}\right\|^{2}+\left\|H_{1}\right\|^{2}\right), t \geq 0 .
$$


This yields the uniqueness, as well as the continuity with respect to the initial data in the $H^{-1} \times L^{2} \times L^{2}$-norm.

It follows from Theorem 3.1 that we can define the family of solving operators

$$
S(t): \Phi \rightarrow \Phi,\left(u_{0}, H_{0}, H_{1}\right) \mapsto\left(u(t), H(t), \frac{\partial H}{\partial t}(t)\right), t \geq 0 .
$$

This family of solving operators forms a semigroup which is continuous with respect to the $H^{-1} \times L^{2} \times L^{2}$-topology and is dissipative in $\Phi$.

Acknowledgments. This paper was initiated while A.M. was visiting the Universitat Politècnica de Catalunya in Terrassa. He wishes to thank this institution for its warm hospitality. R.Q. is supported by the Project "Análisis Matemático de las Ecuaciones en Derivada Parciales de la Termomecánica " (MTM2013-42004-P) of the Spanish Ministry of Economy and Competitiveness.

\section{REFERENCES}

[1] S. Aizicovici, E. Feireisl, Long-time stabilization of solutions to a phase-field model with memory, J. Evol. Eqns. 1 (2001), 69-84.

[2] S. Aizicovici, E. Feireisl, F. Issard-Roch, Long-time convergence of solutions to a phase-field system, Math. Methods Appl. Sci. 24 (2001), 277-287.

[3] D. Brochet, Maximal attractor and inertial sets for some second and fourth order phase field models, Pitman Res. Notes Math. Ser., Vol. 296, Longman Sci. Tech., Harlow, 77-85, 1993.

[4] D. Brochet, X. Chen, D. Hilhorst, Finite dimensional exponential attractors for the phase-field model, Appl. Anal. 49 (1993), 197-212.

[5] D. Brochet, D. Hilhorst, A. Novick-Cohen, Maximal attractor and inertial sets for a conserved phase field model, Adv. Diff. Eqns. 1 (1996), 547-578.

[6] M. Brokate, J. Sprekels, Hysteresis and phase transitions, Springer, New York, 1996.

[7] G. Caginalp, An analysis of a phase field model of a free boundary, Arch. Rational Mech. Anal. 92 (1986), 205-245.

[8] G. Caginalp, Conserved-phase field system: Implications for kinetic undercooling, Phys. Rev. B 38 (1988), 789-791.

[9] G. Caginalp, The dynamics of a conserved phase-field system: Stefan-like, Hele-Shaw and CahnHilliard models as asymptotic limits, IMA J. Appl. Math. 44 (1990), 77-94.

[10] P.J. Chen, M.E. Gurtin, On a theory of heat involving two temperatures, J. Appl. Math. Phys. (ZAMP) 19 (1968), 614-627.

[11] P.J. Chen, M.E. Gurtin, W.O. Williams, A note on non-simple heat conduction, J. Appl. Math. Phys. (ZAMP) 19 (1968), 969-970.

[12] P.J. Chen, M.E. Gurtin, W.O. Williams, On the thermodynamics of non-simple materials with two temperatures, J. Appl. Math. Phys. (ZAMP) 20 (1969), 107-112.

[13] L. Cherfils, A. Miranville, Some results on the asymptotic behavior of the Caginalp system with singular potentials, Adv. Math. Sci. Appl. 17 (2007), 107-129.

[14] L. Cherfils, A. Miranville, On the Caginalp system with dynamic boundary conditions and singular potentials, Appl. Math. 54 (2009), 89-115.

[15] R. Chill, E. Fašangovà, J. Prüss, Convergence to steady states of solutions of the Cahn-Hilliard equation with dynamic boundary conditions, Math. Nachr. 279 (2006), 1448-1462.

[16] M. Conti, S. Gatti, A. Miranville, A generalization of the Caginalp phase-field system with Neumann boundary conditions, Nonlinear Anal. 87 (2013), 11-21. 
[17] C.I. Christov, P.M. Jordan, Heat conduction paradox involving second-sound propagation in moving media, Phys. Review Letters 94 (2005), 154301.

[18] B. Doumbé, Etude de modèles de champ de phase de type Caginalp, PhD thesis, Université de Poitiers, 2013.

[19] A.S. El-Karamany, M.A. Ezzat, On the two-temperature Green-Naghdi thermoelasticity theories, J. Thermal Stresses 34 (2011), 1207-1226.

[20] C.G. Gal, M. Grasselli, The nonisothermal Allen-Cahn equation with dynamic boundary conditions, Discrete Contin. Dyn. Systems A 22 (2008), 1009-1040.

[21] S. Gatti, A. Miranville, Asymptotic behavior of a phase-field system with dynamic boundary conditions, in Differential equations: inverse and direct problems (Proceedings of the workshop "Evolution Equations: Inverse and Direct Problems", Cortona, June 21-25, 2004), A series of Lecture notes in pure and applied mathematics, Vol. 251, A. Favini and A. Lorenzi eds., Chapman \& Hall, 149-170, 2006.

[22] G. Gilardi, On a conserved phase field model with irregular potentials and dynamic boundary conditions, Rend. Cl. Sci. Mat. Nat. 141 (2007), 129-161.

[23] M. Grasselli, A. Miranville, V. Pata, S. Zelik, Well-posedness and long time behavior of a parabolichyperbolic phase-field system with singular potentials, Math. Nachr. 280 (2007), 1475-1509.

[24] M. Grasselli, A. Miranville, G. Schimperna, The Caginalp phase-field system with coupled dynamic boundary conditions and singular potentials, Discrete Contin. Dyn. Systems 28 (2010), 67-98.

[25] M. Grasselli, H. Petzeltová, G. Schimperna, Long time behavior of solutions to the Caginalp system with singular potential, Z. Anal. Anwend. 25 (2006), 51-72.

[26] M. Grasselli, V. Pata, Existence of a universal attractor for a fully hyperbolic phase-field system, J. Evol. Eqns. 4 (2004), 27-51.

[27] A.E. Green, P.M. Naghdi, A re-examination of the basic postulates of thermomechanics, Proc. Royal Society London A 432 (1991), 171-194.

[28] J. Jiang, Convergence to equilibrium for a parabolic-hyperbolic phase-field model with Cattaneo heat flux law, J. Math. Anal. Appl. 341 (2008), 149-169.

[29] J. Jiang, Convergence to equilibrium for a fully hyperbolic phase field model with Cattaneo heat flux law, Math. Methods Appl. Sci. 32 (2009), 1156-1182.

[30] A. Miranville, On a phase-field model with a logarithmic nonlinearity, Appl. Math. 57 (2012), 215229.

[31] A. Miranville, On the conserved phase-field model, J. Math. Anal. Appl. 400 (2013), 143-152.

[32] A. Miranville, A generalized conserved phase-field system based on type III heat conduction, Quart. Appl. Math. LXXI (2013), 755-771.

[33] A. Miranville, Some mathematical models in phase transition, Discrete Contin. Dyn. Systems Series S 7 (2014), 271-306.

[34] A. Miranville, R. Quintanilla, A generalization of the Caginalp phase-field system based on the Cattaneo law, Nonlinear Anal. TMA 71 (2009), 2278-2290.

[35] A. Miranville, R. Quintanilla, Some generalizations of the Caginalp phase-field system, Appl. Anal. 88 (2009), 877-894.

[36] A. Miranville, R. Quintanilla, A phase-field model based on a three-phase-lag heat conduction, Appl. Math. Optim. 63 (2011), 133-150.

[37] A. Miranville, R. Quintanilla, A type III phase-field system with a logarithmic potential, Appl. Math. Letters 24 (2011), 1003-1008.

[38] A. Miranville, R. Quintanilla, A generalization of the Allen-Cahn equation, IMA J. Appl. Math. 80 (2015), 410-430.

[39] A. Miranville, R. Quintanilla, A Caginalp phase-field system based on type III heat conduction with two temperatures, Quart. Appl. Math., to appear.

[40] A. Miranville, S. Zelik, Robust exponential attractors for singularly perturbed phase-field type equations, Electronic J. Diff. Eqns. 2002 (2002), 1-28. 
[41] A. Miranville, S. Zelik, Attractors for dissipative partial differential equations in bounded and unbounded domains, in Handbook of Differential Equations, Evolutionary Partial Differential Equations, Vol. 4, C.M. Dafermos and M. Pokorny eds., Elsevier, Amsterdam, 103-200, 2008.

[42] R. Quintanilla, A well-posed problem for the three-dual-phase-lag heat conduction, J. Thermal Stresses 32 (2009), 1270-1278.

[43] R. Temam, Infinite-dimensional dynamical systems in mechanics and physics, Second edition, Applied Mathematical Sciences, Vol. 68, Springer-Verlag, New York, 1997.

[44] H.M. Youssef, Theory of two-temperature-generalized thermoelasticity, IMA J. Appl. Math. 71 (2006), 383-390.

[45] Z. Zhang, Asymptotic behavior of solutions to the phase-field equations with Neumann boundary conditions, Commun. Pure Appl. Anal. 4 (2005), 683-693.

${ }^{1}$ Université DE Poitiers

Laboratoire de Mathématiques et Applications

UMR CNRS 7348 - SP2MI

Boulevard Marie et Pierre Curie - Téléport 2

F-86962 Chasseneuil Futuroscope Cedex, France

E-mail address: Alain.Miranville@math.univ-poitiers.fr

${ }^{2}$ ESEIAAT-UPC

Departament de Matemàtiques

Colom 11

S-08222 Terrassa, Barcelona, Spain

E-mail address: Ramon.Quintanilla@upc.edu 